\title{
Detección de atrapamiento aéreo en pacientes con sospecha clínica de Enfermedad Pulmonar Obstructiva Crónica y espirometría normal
}

\author{
Detection of air trapping in patients with clinical suspicion \\ of Chronic Obstructive Pulmonary Disease and normal \\ spirometry
}

Darío Londoño MD., MSc. ${ }^{(1)}$; Patricia Hidalgo MD. ${ }^{(2)}$; Isabel Palacios-Ortega, MD. ${ }^{(3)}$

\section{Resumen}

OBJETIVOS: determinar cambios tanto en volúmenes pulmonares como en oscilometría en pacientes sintomáticos con historia exposicional y clasificados por espirometría como sanos o con obstrucción leve, con el fin de corroborar si existe correlación entre la clínica, la pletismografía y la oscilometría con la espirometría en el diagnóstico de obstrucción y si es posible realizar diagnóstico temprano de la EPOC mediante pletismografía y oscilometría, en pacientes con sospecha clínica de la enfermedad.

MATERIALES Y MÉTODOS: se realizó un estudio de correlación, en el que se recolectaron 214 pacientes con sospecha clínica de EPOC, del Hospital Universitario San Ignacio en Bogotá, Colombia, durante el año 2010. Se hicieron cuestionario de síntomas, espirometría, volúmenes pulmonares y oscilometría de impulso.

RESULTADOS: se recolectaron 214 pacientes con sospecha clínica de EPOC, de los cuales el $27 \%$ tuvo espirometría compatible con obstrucción. En los volúmenes pulmonares, el $87 \%$ mostró volumen residual elevado compatible con atrapamiento de aire y el 69\% mostró capacidad funcional residual elevada con relación a hiperinflación. Se midieron resistencias por oscilometría, y el 22\% tuvo resistencias elevadas. La correlación mostró ser muy baja entre espirometría y volúmenes pulmonares así como con resistencias.

CONCLUSIÓN: los pacientes con diagnóstico clínico de EPOC pueden mostrar, de manera temprana, atrapamiento de aire incluso antes de la aparición de anormalidades en la espirometría.

Palabras clave: obstrucción, atrapamiento de aire, volúmenes pulmonares, oscilometría.

\begin{abstract}
OBJECTIVES: to determine changes in lung volumes and oscillometry in symptomatic patients with a history of exposure and classified by spirometry as normal or with slight obstruction, with the aim of establishing whether there is a correlation between clinical findings, plethysmography, and oscillometry with spirometry in diagnosing obstruction, and whether it is possible to achieve early diagnosis of COPD by plethysmography and oscillometry in patients with clinical suspicion of the disease.
\end{abstract}

MATERIALS AND METHODS: a correlation study was carried out, in which 214 patients with clinically suspected COPD from San Ignacio University Hospital (Bogota, Colombia) were collected during the year 2010. A questionnaire of symptoms, spirometry, lung volume measurements, and impulse oscillometry were carried out.

RESULTS: 214 patients with clinically suspected COPD were collected, 27\% of whom

\footnotetext{
${ }^{(1)}$ Internista Neumólogo. Jefe Médico Unidad Neumología

Hospital Universitario San Ignacio. Profesor Asociado Pontificia Universidad Javeriana. Bogotá, Colombia.

${ }^{(2)}$ Internista Neumóloga. Hospital Universitario San Ignacio.

Profesor Asistente Pontificia Universidad Javeriana. Bogotá, Colombia.

${ }^{(3)}$ Internista Neumóloga. Clínica Imbanaco Cali, Colombia.

Correspondencia: Darío Londoño, MD MSc. Correo electrónico: dario.londono@ gmail.com

Recibido: 15-01-2014. Aceptado: 21-03-2014
} 
had spirometry consistent with obstruction. With regard to lung volumes, $87 \%$ had increased residual capacity consistent with air entrapment, and $69 \%$ showed increased residual functional capacity with regard to hyperinflation. Resistances were measured by oscillometry, and $22 \%$ had increased resistances. The correlation between spirometry and lung volumes was very low, as was also the case with resistances.

CONCLUSION: it is possible to find air entrapment early in patients with clinically diagnosed COPD, even before the appearance of spirometric abnormalities.

Keywords: obstruction, air entrapment, lung volumes, oscillometry.

\section{Introducción}

La enfermedad pulmonar obstructiva crónica (EPOC), es una enfermedad prevalente en el mundo, especialmente con el incremento del hábito tabáquico; sin embargo, los datos conocidos de prevalencia varían según la población estudiada y en especial el método de diagnóstico utilizado; de hecho, en la práctica clínica diaria no es infrecuente encontrar pacientes con historia de consumo de cigarrillo y sospecha clínica de EPOC que no cumplen con los criterios diagnósticos espirométricos (1) según GOLD, lo que los aleja del beneficio de un manejo y seguimiento oportunos.

El método universalmente aceptado para la detección de la obstrucción de la vía aérea ha sido la espirometría (2), y de hecho uno de sus componentes, el $\mathrm{VEF}_{1}$, ha sido considerado como marcador pronóstico y de la progresión de la enfermedad desde el estudio publicado por Fletcher (3). No obstante, hoy en la era de los fenotipos de EPOC, se entiende que no todos los pacientes cumplen con las mismas características, no todos muestran el mismo compromiso del $\mathrm{VEF}_{1}$, e incluso en la práctica clínica diaria no es infrecuente encontrar pacientes significativamente sintomáticos con compromiso de su calidad de vida a pesar de tener un espirometría reportada como normal. Esto último ha planteado la posibilidad de que ocurren cambios fisiopatológicos debidos a la EPOC incluso antes de que puedan documentarse alteraciones en el $\mathrm{VEF}_{1}$ (4), generándoles síntomas. Al respecto Meckler ya habría hablado de la importancia de otros hallazgos en las pruebas de función pulmonar en los pacientes con EPOC

\section{¿Qué se conocía previamente del tema?}

La espirometría es la herramienta aceptada para el diagnóstico de la EPOC. Sin embargo, en la práctica clínica no es infrecuente encontrar pacientes con alta sospecha clínica de la enfermedad (factor de riesgo y síntomas) y espirometría normal. Varios trabajos han reportado la utilidad de la medición de resistencias por oscilometría de impulso y volúmenes pulmonares en el diagnóstico de la EPOC, con hallazgos contradictorios.

\section{¿Qué aporta el estudio?}

La frecuencia de alteración de las mediciones de volúmenes pulmonares fue alta en sujetos con espirometría normal y alta sospecha de EPOC (factor de riesgo y síntomas), mientras que no fue así en el caso de las mediciones de oscilometría. La correlación entre espirometría y volúmenes pulmonares y resistencias fue baja. Se requieren estudios adicionales para establecer el valor de mediciones adicionales a la espirometría en el diagnóstico de la EPOC.

que podrían tener importantes implicaciones terapéuticas, entre ellos el volumen residual (VR), cuya elevación se consideró posteriormente como la causante de la reducción del $\mathrm{VEF}_{1}$, y por tanto el atrapamiento de aire como una manifestación temprana de EPOC y un blanco terapéutico interesante, siendo entonces el VR un posible nuevo "biomarcador" en dicha enfermedad (5).

Bajo el precepto de que existen cambios fisiopatológicos tempranos en la EPOC que se demuestran en volúmenes pulmonares antes de documentarse en espirometría, se llevó a cabo este estudio en el cual se evaluaron pacientes con sospecha clínica de EPOC a quienes se les practicaron tanto espirometría como volúmenes pulmonares por pletismografía y oscilometría de impulso con 
el fin de demostrar que es posible encontrar pacientes sintomáticos que por espirometría se habrían clasificado como sanos o con obstrucción leve, con importantes alteraciones documentadas en los volúmenes pulmonares, especialmente en volumen residual, capacidad pulmonar total, capacidad funcional residual, así como en la medición de resistencias pulmonares.

\section{Objetivos}

El estudio busca determinar si existe correlación entre los datos obtenidos a partir de la pletismografía y la oscilometría de impulso, con aquellos generados por espirometría para el diagnóstico de obstrucción bronquial en pacientes con sospecha clínica de EPOC. Así mismo, se desea determinar si es posible realizar diagnóstico temprano de EPOC mediante pletismografía y oscilometría, en pacientes con diagnóstico clínico de esta y con espirometría normal, así como establecer la correlación entre espirometría y volúmenes pulmonares y entre espirometría y resistencias por oscilometría y finalmente el número de sujetos diagnosticados con EPOC por espirometría, volúmenes pulmonares y por resistencias.

\section{Materiales y métodos}

Se realizó un estudio de correlación entre criterios clínicos, espirometría, volúmenes pulmonares y resistencias por oscilometría, para lo cual se incluyeron 214 pacientes adultos que consultaron a la unidad de neumología del Hospital Universitario San Ignacio, de Bogotá, detectados por cuestionario de síntomas respiratorios con claros factores exposicionales tanto al cigarrillo, como al humo de leña y/o ocupacional, de tal manera que se incluyeran en el estudio pacientes con alta sospecha de padecer EPOC, durante el año 2010.

Se excluyeron aquellos con diagnóstico de asma y aquellos con exacerbación aguda de la EPOC en el momento del estudio.

A todos se les aplicó un cuestionario de síntomas especialmente diseñado para detectar pacientes con sospecha clínica y antecedente exposicional. A todos se les realizaron mediciones de peso, talla, espirometría, curva flujo volumen, volúmenes pulmonares por pletismografía y oscilometría de impulso, todos teniendo en cuenta la técnica y los valores de referencia de la ATS $(6,7)$. Para efectuar las pruebas de función pulmonar se usó un equipo Jaeger, Jaeger Master Screen IOS y Jaeger Master Screen Body.

Para la correlación se utilizó el coeficiente de correlación de Pearson, el cual reflejaba la tendencia entre los resultados de los métodos evaluados.

\section{Resultados}

En el estudio se incluyeron 214 pacientes adultos, 87 hombres y 127 mujeres, cuyas edades oscilaban entre los 45 y los 86 años. El 100\% tenía sospecha clínica de EPOC, dada por disnea, tos seca o con expectoración crónica, y algún tipo de antecedente exposicional bien fuera humo de leña, cigarrillo u ocupacional.

El $100 \%$ de los pacientes incluidos tenía alta sospecha clínica de EPOC; 59 (27\%) mostraron espirometría compatible con alteración obstructiva (relación $\left.\mathrm{VEF}_{1} / \mathrm{CVF}<0,7\right)$ y los 155 restantes tenían espirometría normal (72,4\%). 12 de 214 tenían una espirometría compatible con alteración restrictiva, pero al llevar a estos mismos pacientes a realización de volúmenes pulmonares no se documentó tal alteración.

En los volúmenes pulmonares por pletismografía, 186 pacientes, el $87 \%$ de los 214 encuestados, mostraron volumen residual (VR) considerado como anormalmente elevado $(>120 \%)$; en capacidad funcional residual (CFR), se encontraron 147 pacientes $(68,6 \%)$ con valores elevados (elevado con más de $120 \%$ del predicho). Los valores de capacidad pulmonar total (CPT) se encontraron elevados en 132 pacientes (61\%). No se documentaron pacientes con CPT disminuida que los definiera como restrictivos (Figuras 1 y 2).

Al mismo tiempo, se realizó medición de resistencias de la vía aérea por oscilometría de impulso 


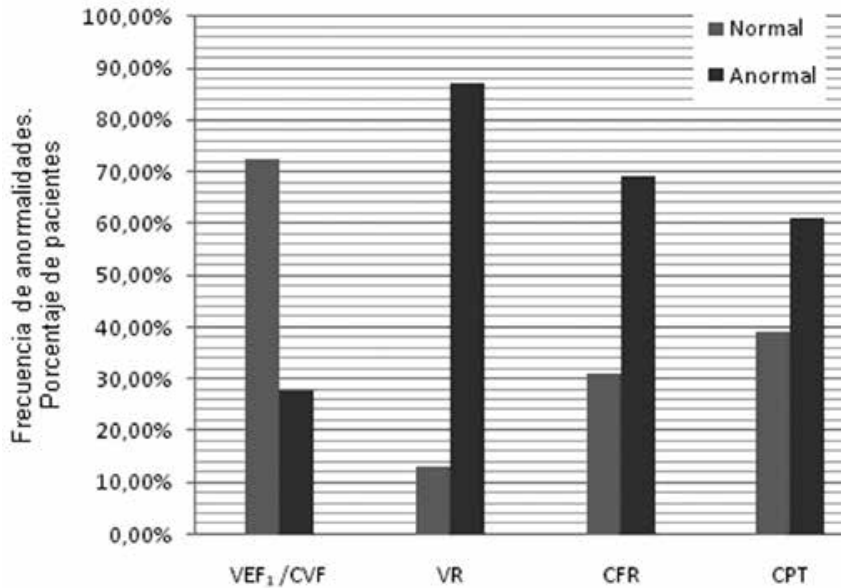

Figura 1. Porcentaje de pacientes con función pulmonar anormal de toda la población general. Se consideraban anormales si VEF, /CVF $<0,7$, VR $>120 \%$ del predicho, CFR $>120 \%$ del predicho o CPT $>120 \%$ del predicho.

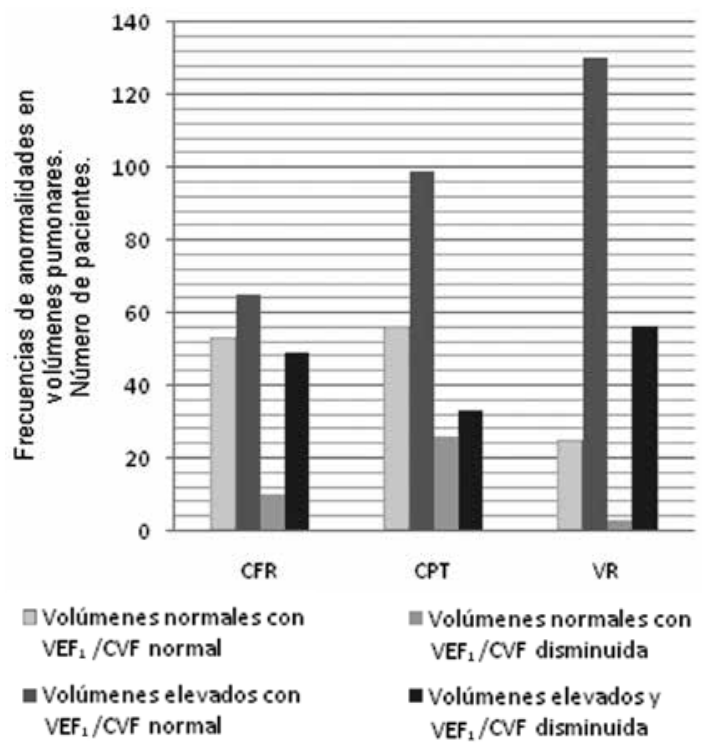

Figura 2. Frecuencia de anormalidades en volúmenes pulmonares en pacientes con espirometría normal y anormal.

buscando encontrar si estas podrían ayudar a documentar obstrucción en pacientes sintomáticos; 48 pacientes mostraron resistencias elevadas a $5 \mathrm{~Hz}(22 \%), 166$ de los 214 aún estando sintomáticos mostraron resistencias consideradas como normales $(78 \%)$. De los pacientes con resistencias normales, el 79\% de ellos tuvieron espirometría normal; $21 \%$ de ellos tenían espirometría obstructiva. Los volúmenes pulmonares en pacientes con resistencias normales mostraron: $76 \%$ con CFR elevada, $65 \%$ con CPT elevada y $86 \%$ VR elevado. En pacientes con resistencias anormalmente elevadas, la espirometría mostró patrón obstructivo en $50 \%$ de ellos, por otro lado, en cuanto a volúmenes en pacientes con resistencias elevadas, $50 \%, 48 \%$ y $88 \%$ mostraron CFR, CPT y VR elevados, respectivamente.

Al final del estudio se pudo hacer diagnóstico de EPOC en 59 pacientes con espirometría anormal, 27,5\%; por volúmenes pulmonares en 186 pacientes, $87 \%$; y en 48 pacientes por oscilometría de impulso, $22 \%$.

Al realizar la correlación entre espirometría y volúmenes pulmonares, así como con resistencias medidas por oscilometría, los resultados mostraron una correlación entre $\mathrm{VEF}_{1}$ y CFR de 0,07 con índice de correlación de Pearson de -0,26. Entre $\mathrm{VEF}_{1}$ y VR, la correlación fue de 0,12 , con un índice de Pearson de -0,35 (Figura 3), mientras que la correlación entre $\mathrm{VEF}_{1}$ y CPT fue de 0,059 con un índice Pearson de 0,24 . Cuando se estudió $\mathrm{VEF}_{1}$ con resistencias a $5 \mathrm{~Hz}$, la correlación fue de 0,12 con un índice de Pearson de -0,35. Igualmente la correlación entre $\mathrm{VEF}_{1} / \mathrm{CVF}$ con VR, mostró correlación 0,2 , índice de Pearson - 0,44 ; la correlación $\mathrm{VEF}_{1} / \mathrm{CVF}$ con CFR fue de 0,22, índice de Pearson de -0,47; la

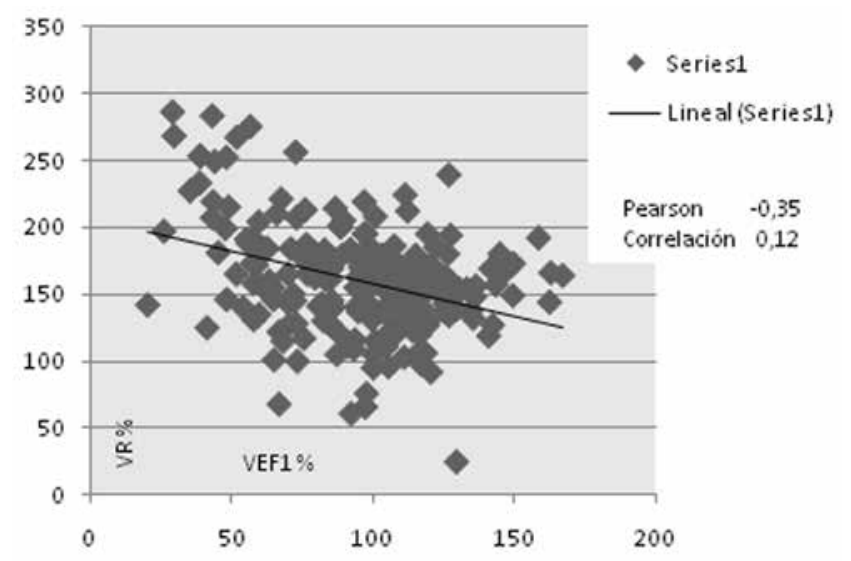

Figura 3. Correlación $\mathrm{VEF}_{1}$ (porcentaje del predicho) y volumen residual (porcentaje del predicho). 
correlación $\mathrm{VEF}_{1} / \mathrm{CVF}$ con $\mathrm{CPT} 0,006$ y un índice de correlación de $-0,079$ y la correlación $\mathrm{VEF}_{1} / \mathrm{CVF}$ con resistencias a $5 \mathrm{~Hz}$ fue de 0,12 , con índice de Pearson de $-0,35$ (Figuras 4 a 7).

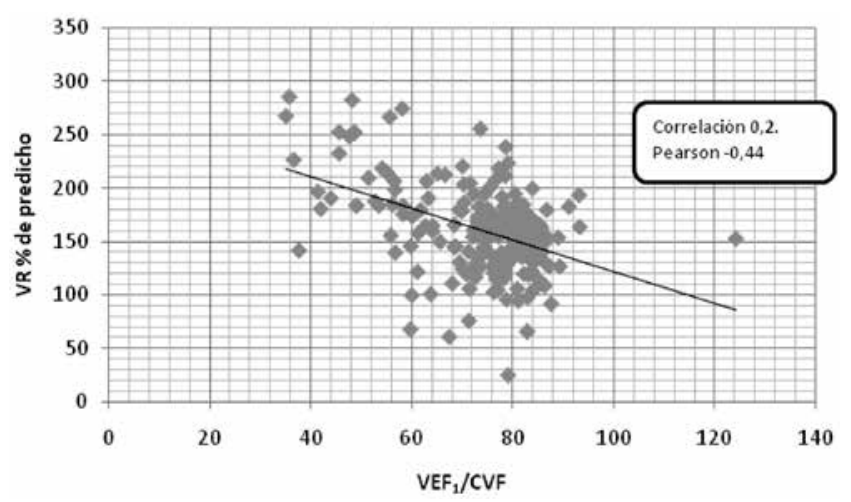

Figura 4. Correlación entre $\mathrm{VEF}_{1} / \mathrm{CVF}$ y volumen residual (VR).

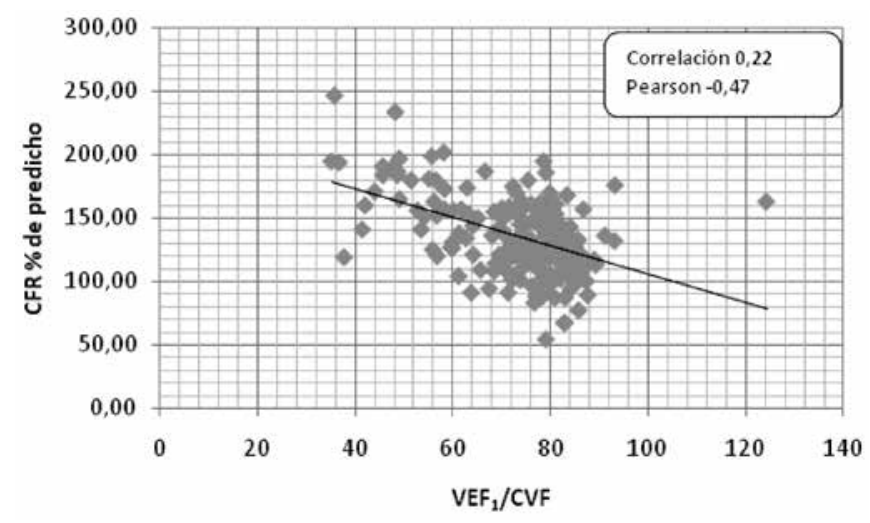

Figura 5. Correlación $\mathrm{VEF}_{1} / \mathrm{CVF}$ con capacidad funcional residual (CFR).

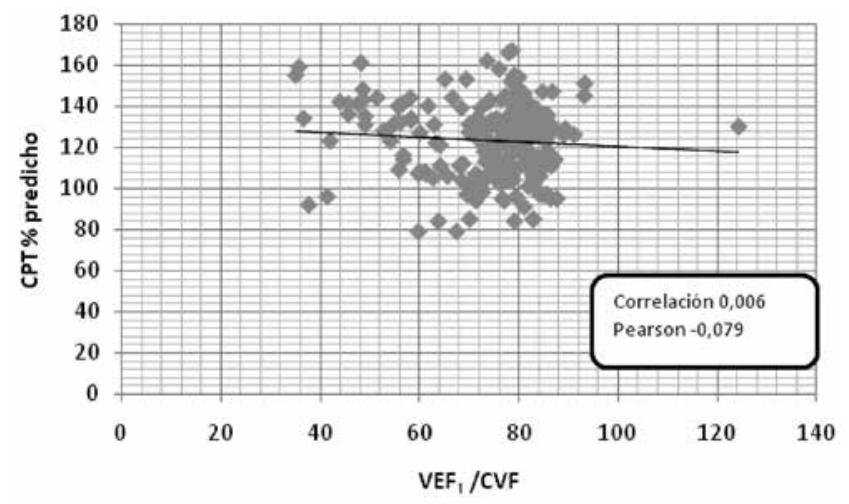

Figura 6. Correlación VEF $/$ /CVF y capacidad pulmonar total (CPT) (porcentaje del predicho).

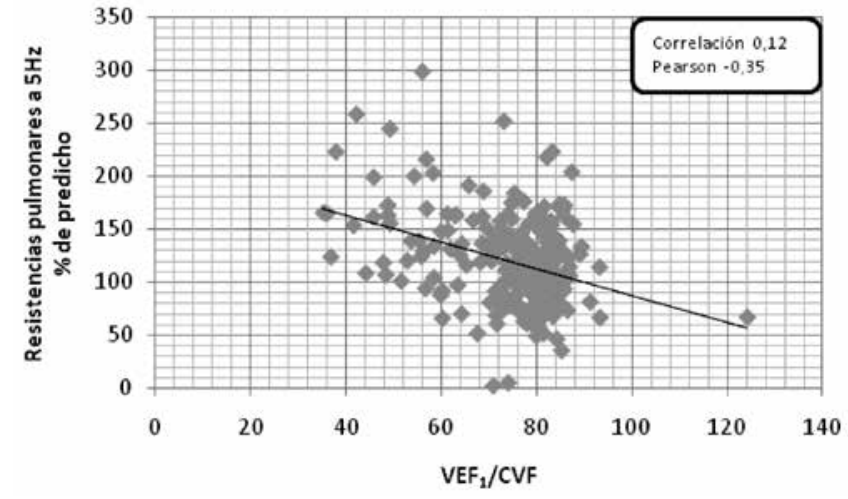

Figura 7. Correlación $\mathrm{VEF}_{1} / \mathrm{CVF}$ y resistencias pulmonares a $5 \mathrm{~Hz}$ (porcentaje del predicho).

\section{Discusión}

La espirometría es el método mundialmente aceptado para el diagnóstico de la EPOC, y ha sido avalado por las guías de manejo (1); sin embargo, no es infrecuente encontrar pacientes con alta sospecha clínica de EPOC pero con espirometría normal, fenómeno que apoya el concepto ya reconocido de que no es posible diagnosticar todos los pacientes con EPOC con el mismo método diagnóstico. Así mismo, estudios previos habrían mostrado por tanto que la detección de la EPOC y por tanto su prevalencia, dependen en parte del método diagnóstico utilizado (8), y al que con más frecuencia se recurre es a la espirometría. De lo anterior, sumado a que es claro que existen hallazgos fisiopatológicos tempranos incluso antes de documentarlos en la espirometría, nace la idea de complementar el diagnóstico con pruebas como los volúmenes pulmonares por pletismografía y tal vez la oscilometría de impulso. Muestra de ese subdiagnóstico de la EPOC mediante espirometría, son los resultados de este estudio en pacientes con diagnóstico clínico de EPOC con antecedente exposicional, en quienes pese a sus síntomas, el $72,4 \%$ de la muestra tuvieron espirometría normal, poniendo en duda el diagnóstico por este método.

Aparecen entonces hallazgos relevantes por pletismografía, a diferencia de lo encontrado en espirometría; la mayoría de los pacientes con sospecha clínica tenían anormalidades tanto en CFR como en CPT y especialmente en VR, al igual que el $87 \%$ mostraron algún grado de atrapamiento de aire por 
elevación del VR. Con lo anterior es posible proponer los volúmenes pulmonares como un método diagnóstico de la EPOC especialmente en aquellos pacientes cuya espirometría es normal pero persiste la sospecha clínica; por tanto, sería posible realizar más diagnósticos de EPOC y más pacientes se beneficiarían del seguimiento y terapia apropiados.

Existen múltiples estudios que han intentado demostrar la utilidad de medición de resistencias por oscilometría de impulso $(9,10)$ en el diagnóstico de la EPOC; sin embargo, los hallazgos han sido contradictorios, pero es un método útil en especial en quienes se dificulta realizar las maniobras requeridas para la realización de espirometría y volúmenes pulmonares. En este estudio, con la intención de ofrecer una alternativa diagnóstica en la EPOC, se llevaron a cabo mediciones de resistencias mediante oscilometría de impulso a todos los pacientes. Los resultados continúan generando incertidumbre respecto a este método ya que al igual que con la espirometría la mayoría de pacientes mostró resistencias normales; sólo el $22 \%$ tuvo resistencias elevadas. Por otro lado, se evaluó si estos eran los mismos pacientes que mostraron alteración en la espirometría, y al respecto no se encontraron resultados positivos ya que sólo la mitad mostró relación $\mathrm{VEF}_{1} / \mathrm{CVF}$ disminuida, y finalmente, no se documentó correlación entre espirometría y resistencias de la vía aérea a $5 \mathrm{~Hz}$ (la correlación fue de 0,12 con un índice de Pearson de -0,35). A pesar del anterior hallazgo, cuando se compararon resistencias y volumen residual, el $87 \%$ de los pacientes con resistencias anormales igualmente tenía volumen residual elevado, lo que sigue aportando sustento al uso de los volúmenes pulmonares.

\section{Conclusiones}

La espirometría continúa siendo un método importante en la detección de pacientes con sospecha clínica de EPOC, no obstante, bien se sabe que el primer cambio fisiopatológico en estos pacientes es la elevación del VR incluso antes de documentarse una alteración en la espirometría. En el estudio se incluyeron pacientes con alta sospecha clínica de EPOC con antecedente de exposición de riesgo, y si bien la mayoría tenía espirometría normal ya tenían cambios representados en los volúmenes pulmonares por pletismografía en especial en elevación del VR. Con lo anterior, la medición de volúmenes pulmonares es una alternativa diagnóstica importante, específicamente en pacientes cuya sospecha clínica es alta a pesar de una espirometría aparentemente normal.

Respecto a la medición de resistencias por oscilometría, es una buena opción diagnóstica para quienes es difícil realizar las maniobras relacionadas con la espirometría; sin embargo, el hecho de que la mayoría de los pacientes parecieran normales por este método, hace pensar que no es del todo útil en aquellos con alta sospecha clínica y espirometría normal.

\section{Agradecimientos}

Al personal de la Unidad de Neumología del Hospital Universitario San Ignacio, Bogotá Colombia.

\section{Bibliografía}

1. Global Strategy for the Diagnosis, Management and Prevention of COPD, Global Initiative for Chronic Obstructive Lung Disease (GOLD) 2011. Disponible en: http://www.goldcopd.org/.

2. García Río F, Lores V, Rojo B. Evaluación funcional respiratoria (obstrucción y atrapamiento). Arch Bronconeumol. 2007;43 (Supl 3): 8-14.

3. Fletcher C, Peto R. The natural history of chronic airflow obstruction. Br Med J. 1977;1:1645-1648.

4. Macklem PT. Therapeutic implications of the pathophysiology of COPD. Eur Respir J. 2010;35:676-680.

5. Deesomchok A, Webb KA, Forkert L, Lam YM, Ofir D, Jensen D, O'Donnell DE. Lung hyperinflation and its reversibility in patients with airway obstruction of varying severity. COPD. 2010;7:428-37.

6. Miller MR, Hankinson J, Brusasco V, Burgos F, Casaburi R, Coates A, et al. ATS/ERS Task Force. Standardisation of spirometry. Eur Respir J. 2005;26:319-38.

7. Oostveen E, MacLeod D, Lorino H, Farré R, Hantos Z, Desager K, Marchal F; ERS Task Force on Respiratory Impedance Measurements. The forced oscillation technique in clinical practice: methodology, recommendations and future developments. Eur Respir J. 2003;22:1026-41.

8. Halbert RJ, Isonaka S, George D, Iqbal A. Interpreting COPD prevalence estimates: what is the true burden of disease? Chest. 2003;123:1684-92.

9. Kolsum U, Borrill Z, Roy K, Starkey C, Vestbo J, Houghton C, Singh D. Impulse oscillometry in COPD: identification of measurements related to airway obstruction, airway conductance and lung volumes. Respir Med. 2009;103:136-43.

10. Crim C, Celli B, Edwards LD, Wouters E, Coxson HO, Tal-Singer R, Calverley PM; ECLIPSE investigators. Respiratory system impedance with impulse oscillometry in healthy and COPD subjects: ECLIPSE baseline results. Respir Med. 2011;105:1069-78. 